\title{
Acute Effects of Whole-Body Vibration on Dopplerfluxometry of the Common Carotid Artery Parameters among Adult and Elderly Non-Athletes Healthy Dogs
}

\author{
Lais Rosa Nagai', Sheila Canevese Rahal', Carmel Dadalto', Bruna Martins da Silva', \\ Miriam Tsunemi2 ${ }^{2}$, Maria Jaqueline Mamprim', Marina Frazatti Gallina', \\ Ygor Faria Nagano', Stella Helena Sakata Lopes' \& Ivan Felismino Charas dos Santos ${ }^{1}$
}

\begin{abstract}
Background: Whole-Body Vibration (WBV) is an oscillatory mechanical stimulus spreading throughout the body and considered a type of physical exercise because of the activation of the cardiovascular, musculoskeletal, and neuroendocrine systems. It is a physical exercise modality since it promotes cardiovascular resistance, increase in muscular strength and neurosensitivity, and motor coordination improvement. For use of WBV as an exercise modality for dogs, it is necessary to evaluate the Dopplerfluxometry parameters of the common carotid artery in healthy dogs in order to perform a safe protocol without inducing any cerebral alteration. This study aimed to evaluate the acute effects of WBV on systolic peak velocity (SPV), resistivity (RI), and pulsatility index (PI) of the both common carotid artery among adults and elderly non-athletes healthy dog.

Materials, Methods \& Results: Fourteen clinically healthy, neutered crossbreed male dogs, non-athlete were divided into two groups of seven dogs, according to the age group: Group 1 (G1)- Adult dogs: age between 12 and 84 months; Group 2 (G2)- Elderly dogs: aged over 84 months. All dogs were submitted to sessions of WBV using the protocol of $30 \mathrm{~Hz}$ for 5 min, followed by an increase to $50 \mathrm{~Hz}$ for 5 more min and ending with $5 \mathrm{~min}$ at $30 \mathrm{~Hz}$, without rest between the variation of the vibration frequency. The systolic peak velocity (SPV), resistivity (RI), and pulsatility index (PI) of the common carotid artery were assessed in two time-points: 5 min before the WBV sessions (5PRE) and 1 min after the WBV (1POS). No significant variations in the SPS, RI, and PI of both common carotid artery of the G1 and G2 were identified. The anatomic reference for the left and right common carotid artery was the right and left extern jugulars veins, which were identified by the venous blood vessel characteristics as endothelium type, and single-phase without systolic peaks wave. Discussion: In humans, WBV is indicated to muscle size and tone increase, therefore it is believed that this modality can present a beneficial result in dogs with muscular atrophy associated with orthopedic surgeries and in cases of osteoarthritis. Irreversible brain damage can be caused by a possible hemodynamic alteration in the common carotid arteries resulted from mechanical vibrations. Parkinson's disease patients and individuals affected by strokes that went under acute sessions of WBV showed handshake reduction and better proprioception, respectively. The beneficial effect was observed in cervix opening in dogs with metritis. The behavior of sitting of the dogs over $30 \mathrm{~kg}$ during WBVsessions was associated with paraparesis. This result was present in medium-size non-athletic dogs weighing from 10.1 to $17.9 \mathrm{~kg}$ that went through WBV for 5 consecutive days, using the same vibrating platform. No signs of discomfort during a single session of $10 \mathrm{~min}$ of WBV (15 and $21 \mathrm{~Hz})$ were observed in healthy adult horses. Studies using mechanical vibrations on adult healthy dogs did not show significant variation RI of the renal artery. On the other hand, daily use of WBV for 5 days on dogs showed significant enhancement on RI of the femoral artery immediately after it. A single session of WBV $(30$ and $50 \mathrm{~Hz}$ did, for $15 \mathrm{~min}$ ) do not produce undesirable effects on SPV, RI, and PI of both common carotid arteries in adult and elderly non-athletic healthy dogs.
\end{abstract}

Keywords: exercise, mechanic vibration, vibrating platform, resistivity index, systolic peak velocity.

${ }^{1}$ Faculdade de Medicina Veterinária e Zootecnia (FMVZ) \& ${ }^{2}$ Instituto de Biociências (IBB), Universidade Estadual Paulista (Unesp), Botucatu, SP, Brazil. CORRESPONDENCE: I.F.C. Santos [ivansantos7@ hotmail.com]. FMVZ - UNESP Campus de Botucatu. Av. Prof. Doutor Walter Mauricio Correa s/n. CEP 18618-681 Botucatu, SP, Brazil. 


\section{INTRODUCTION}

Whole-Body Vibration (WBV) is a vibratory mechanic stimulus induced by a vibrating platform. This vibration propagates through the body, activating the cardiovascular, musculoskeletal, and neuroendocrine systems $[4,6,8]$. It is considered a physical exercise modality since it promotes increasing regional blood-flow and cardiovascular resistance, muscular strength and hypertrophy, neurosensitivity, and motor coordination improvement among others [4,8,18,19,21,29].

Studies regarding WBV effects in dogs were present in the literature with less frequently [10,22-24]. To use the WBV as an exercise modality for dogs, it is necessary to evaluate the Dopplerfluxometry parameters of the common carotid artery in healthy dogs in order to perform a safe protocol without inducing cerebral alterations.

In this context, the present aimed to evaluate the acute effects of WBV on systolic peak velocity (SPV), resistivity (RI), and pulsatility index (PI) of both common carotid artery among adults and elderly non-athletes healthy dog. The hypothesis was that a single session of WBV $(30 \mathrm{~Hz}$ and $50 \mathrm{~Hz})$ during 15 min isn't capable to induce a deleterious effect in the SPV, RI, and PI of both common carotid artery in these animals.

\section{MATERIALS AND METHODS}

\section{Animals selection}

The study was performed at $892 \mathrm{~m}$ of altitude (GPS: -22.888941850000002, -48.49840491716181) during the summer (February - March). The experimental procedures were explained to the dog's owner that signed a form that allows us to use his animal in the study.

Fourteen clinically healthy, neutered crossbreed male dogs, non-athlete were used. All the dogs were considered non-athletic because they stayed most of the time indoors and went for a 20 min daily walk on the leash. According to the owners, the animals were sociable and docile. Their diet consisted of 250 to 300 $\mathrm{g}$ per day of dry food for medium size dogs.

\section{Inclusion criteria}

Dogs with no clinical alteration during the physical exam, neurological and musculoskeletal exams; no changes on the blood cells count and bio- chemical parameters (alanine aminotransferase, aspartate aminotransferase, creatinine, creatinine kinase, total proteins, and albumin); and dogs with a body condition score of three in the 9-point body condition scoring [14].

\section{Exclusion criteria}

Dogs submitted to any surgical procedure or medical treatment at least 30 days before the study; dogs presented cardiac alteration with hemodynamic repercussions diagnosed by echodopplercardiography exam; animals that presented arterial hypertension were excluded from the study.

\section{Experimental design}

The animals were divided into 2 groups of $7 \mathrm{dogs}$, according to the age group: Group 1 (G1)Adult dogs: age between 12 and 84 months; Group 2 (G2)- Elderly dogs: age over 84 months. The body weight $(\mathrm{BW})$ was measured using a digital scale $\left(\text { Amira Ydtech }{ }^{\circledR}\right)^{1}[200 \mathrm{~kg} / 100 \mathrm{~g}, 70 x 50]$. The body length (BL) was determined by the distance between the scapulohumeral joint and the tuberosity of the ischium, and the forelimb height (FLH) by the distance between the ground and the scapula's dorsal border. Both parameters were quantified using a glass fiber body tape (KS Tools Werkzeuge $\left.{ }^{\circledR}\right)^{2}$. For body proportion $(\mathrm{BP})$ calculation, the formula: $\mathrm{BP}=\mathrm{FLH} / \mathrm{BL}$ was used [28].

\section{Whole-body vibration}

Before the WBV sessions, the dogs fasted for $6 \mathrm{~h}$ and were left $2 \mathrm{~h}$ without water. For environmental acclimation and physical rest, dogs stayed on the top of the vibrating platform turned-off one hour before the sessions. All the sessions were performed at the $02: 00 \mathrm{pm}$ at the room with $22^{\circ} \mathrm{C}$ and $40-45 \%$ relative humidity. The room luminosity was controlled by fluorescent lamps and blackout curtains. Four persons were allowed in the room and were avoided to minimize movement and noise.

To perform WBV sessions, a vibrating platform $\left(\text { TheraPlate }{ }^{\circledR}\right)^{3}[$ length $=92.0 \mathrm{~cm}$, width $=62.0$ $\mathrm{cm}$ and height $=16.0 \mathrm{~cm}]$ which produce vortex circulation, and made up for dogs and cats. During the sessions, dogs were prevented of sitting and laying down by using a leash (Figure 1). The WBV protocol consisted of a $30 \mathrm{~Hz}$ for 5-min (peak-to-peak displacement $=3.10 \mathrm{~mm}$; peak acceleration $=11.16$ 
$\mathrm{m} / \mathrm{s}^{2}$; peak velocity $\left.=0.29 \mathrm{~m} / \mathrm{s}\right)$, followed by $50 \mathrm{~Hz}$ for 5-min (peak-to-peak displacement $=3.98 \mathrm{~mm}$; peak acceleration $=39.75 \mathrm{~m} / \mathrm{s}^{2}$; peak velocity $=0.62$ $\mathrm{m} / \mathrm{s}$ ), and ending with $30 \mathrm{~Hz}$ for 5 -min (peak-to-peak displacement $=3.10 \mathrm{~mm}$; peak acceleration $=11.16$ $\mathrm{m} / \mathrm{s}^{2}$; peak velocity $=0.29 \mathrm{~m} / \mathrm{s}$ ) without rest between the frequencies.

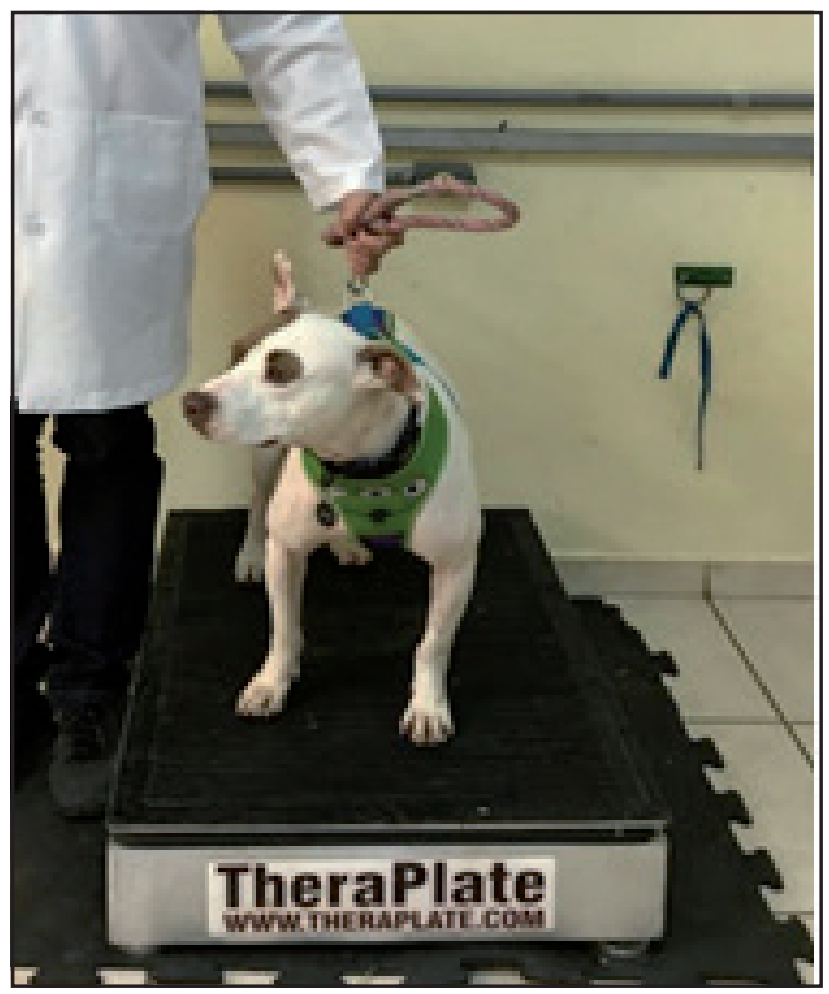

Figure 1. Dog being restrained by a leash in the center of the vibrating platform $\left(\right.$ TheraPlate $\left.{ }^{\circledR}\right)[$ length $=92.0 \mathrm{~cm}$, width $=62.0 \mathrm{~cm}$ and height $=16.0 \mathrm{~cm}]$.

The platform's frequencies were verified by a digital oscilloscope, the acceleration peak (Apeak) by an accelerometer and the peak-to-peak displacement (Dpeak) by using the formula: Dpeak $=$ Apeak $/(2 . f)^{2}$ (f - vibration frequency) and peak velocity (Vpeak ) by using the formula: Vpeak $=$ Dpeak $\pi . f(\pi=3,14)$ $[6,21]$.

The dog's behavior included gastrointestinal (emesis and diarrhea) alterations that were observed within $24 \mathrm{~h}$ after the WBV sessions. The first $3 \mathrm{~h}$ were performed by an independent researcher and the others 21 by the owners in their houses.

\section{Dopplerfluxometry evaluation}

The Dopplerfluxometry evaluation of the common carotid artery was performed with the animal standing position on the vibrating platform turned-off in two different time-points: 5-min before the WBV session (5PRE) and 1-min after the WBV (1POST).

The anatomic reference for the left and right common carotid artery was the right and left extern jugulars veins, identified by the venous blood vessel characteristics (superficiality, thin endothelium, and single-phase without systolic peaks wave). Dopplerfluxometry was performed by using a spectral Doppler after arterial identification, using ultrasonography equipment (MyLab Alpha Esaote $\left.{ }^{\circledR}\right)^{4}$ and a linear transducer $(10 \mathrm{MHz})$. The sonograms were performed in transversal and longitudinal views, caudal to the carotid bifurcation.

The angle of the ultrasound beam with the blood flow was standardized at $20^{\circ}$, and the acoustic gel was used between the transducer and the dog's skin. All exams were done by the same person, with experience in ultrasonography exams.

The SPV, RI, and PI of both common carotid arteries were the parameters evaluated in the Dopplerfluxometry exam. These parameters were calculated by the software of the ultrasound equipment, however, to determine the SPV the three measures' average were used (Figure 2).

\section{Statistical analysis}

Difference between the SPV, RI, and PI values in the evaluated time-points were detected by ANOVA followed by the Tukey test. Values were expressed as average \pm standard deviation. Differences with $P<$ 0.05 were considered significant. Statistical analysis was made using GraphPad Instat ${ }^{\circledR 5}$.

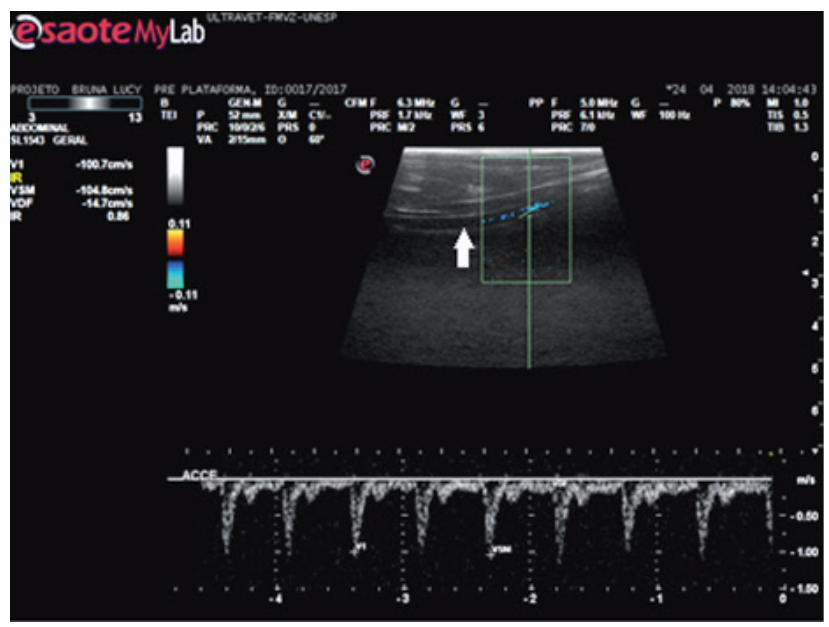

Figure 2. Sonogram with a longitudinal view showing the left common carotid artery (white arrow) to determinate the SPV, RI, and PI in an adult healthy dog, before the WBV session. 


\section{RESULTS}

The age, BW, BL, and FLH parametrs of dogs in both groups were shown in table 1. All animals had a BP below 1, being considered long body or short limbs dogs. It was not necessary to sedate or training the dogs to use the vibrating platform. The animals remained in standing position during WBV session, however, 8-min after the WBV beginning, the dogs above $30 \mathrm{~kg}$ tried to sit. After the session, all dogs remained agitated for approximately 5 -min, followed by a calm behavior for the next $12 \mathrm{~h}$. No gastrointestinal alteration, such as diarrhea and emesis, was noticed.

No significant variation was observed in SPV, RI, and PI values of the left (LCCA) and the right common carotid artery (RCCA) in all time-points (Table 2).

Table 1. Mean and standard deviation $(\mathrm{Me} \pm \mathrm{SD})$ of age, BW, BL and FLH of dogs of G1 and G2 groups.

\begin{tabular}{ccc}
\hline Parameter & G1 $(\mathrm{n}=7)$ & G2 $(\mathrm{n}=7)$ \\
\hline Age (months) & $27.40 \pm 5.90$ & $96.00 \pm 13.90$ \\
BW (kg) & $22.81 \pm 3.40$ & $27.82 \pm 8.80$ \\
BL (cm) & $66.29 \pm 4.20$ & $76.57 \pm 7.00$ \\
FLH (cm) & $47.29 \pm 4.30$ & $48.41 \pm 5.90$ \\
BP & $0.71 \pm 0.04$ & $0.66 \pm 0.05$ \\
\hline
\end{tabular}

Table 2. Mean and standard deviation $(\mathrm{Me} \pm \mathrm{SD}$ ) of SPV, RI, and PI of the left (LCCA) and right common carotid artery (RCCA) of dogs of G1 and G2 groups at 5PRE and 1POS.

\begin{tabular}{ccccc}
\hline \multirow{2}{*}{ Parameter } & \multicolumn{2}{c}{ 5PRE } & \multicolumn{2}{c}{ 1POS } \\
\cline { 2 - 5 } & G1 $(\mathrm{n}=7)$ & $\mathrm{G} 2(\mathrm{n}=7)$ & $\mathrm{G} 1(\mathrm{n}=7)$ & $\mathrm{G} 2(\mathrm{n}=7)$ \\
\hline SPV $(\mathrm{cm} / \mathrm{s})$ & & & & $59.30 \pm 9.50$ \\
RCCA & $73.30 \pm 24.60$ & $57.50 \pm 10.00$ & $76.50 \pm 19.00$ & $55.00 \pm 8.00$ \\
\hline LCCA & $91.50 \pm 34.50$ & $54.30 \pm 13.70$ & $98.60 \pm 26.60$ & $0.85 \pm 0.06$ \\
\hline RI & & & $0.81 \pm 0.05$ & $0.84 \pm 0.05$ \\
\hline RCCA & $0.86 \pm 0.02$ & $0.87 \pm 0.04$ & $0.80 \pm 0.06$ & $2.50 \pm 0.20$ \\
LCCA & $0.90 \pm 0.03$ & $0.91 \pm 0.04$ & & $2.60 \pm 0.50$ \\
\hline PI & & & $2.20 \pm 0.50$ & $2.00 \pm 0.30$ \\
\hline LCCA & $2.30 \pm 0.40$ & $2.60 \pm 0.40$ & & \\
\hline
\end{tabular}

\section{DISCUSSION}

The hypothesis was confirmed, since the results of the study showed that a single session of WBV of $30 \mathrm{~Hz}$ (5-min) $-50 \mathrm{~Hz}$ (5-min)- $30 \mathrm{~Hz}$ (5-min) did not cause significant alteration on the SPV, RI and PI values of the RCCA and LCCA in adult and elderly healthy non- athlete dogs. The WBV in human patients is indicated for muscle size and tone increase $[3,6,7,15,18$, $20,21]$, therefore it is believed that this modality can present a positive result in dogs with muscular atrophy due to orthopedic surgeries and in osteoarthritis.

The common carotid arteries are responsible to suppress the head with oxygenated blood [2], and possible hemodynamic alterations associated with the mechanic vibration could cause irreversible deleterious to the brain.

Parkinson's disease's patients submitted to acute WBV sessions with 1 to $6 \mathrm{~Hz}$ variation, and 1.5 to
$3.0 \mathrm{~mm}$ peak-to-peak displacement showed handshake and muscular stiffness reduction [11,26]. Therefore, vibratory therapy can be an alternative in the initial phase of degenerative diseases [21]. Patients affected by cerebral ischemia and submitted to WBV $(30 \mathrm{~Hz}$, $3.0 \mathrm{~mm}$ peak-to-peak displacement) as rehabilitation modality, showed increased proprioception and better posture [27].

The WBV protocol used in this study was following previous studies using medium size dogs $[10,23,24]$ since there are no other security protocols for dogs. The single session of WBV had benefits related to the cervix opening in female dogs diagnosed with metritis [22]. However, harmful effects were noticed in professional and amateur athletes $[9,13,17]$.

The dogs from both groups were classified with long body conformation or short limbs dogs, since the $\mathrm{BP}$ was lower than 1 [28], conceding a body uniformity 
of dogs used in this study. Considering the influence of the peak-to-peak displacement in a different body, the body conformation uniformity needs to be considered when WBV is performed [6].

All dogs fasted since there are different gastrointestinal motility responses in humans' patients submitted in different WBV protocols $[12,16]$. The standing position sure the uniformity of distribution of the vibratory stimulus [3,6,19-21].

Dogs over $30 \mathrm{~kg}$ tried to sit during the WBV and was associated with paraparesis or loss sensibility of the limbs due to higher pressure on the plantar/palmar region. This behavior was observed in a mediumsized non-athletic healthy dog weighing from 10.1 to $17.9 \mathrm{~kg}$ that submitted to WBV for 5 consecutive days, using the same protocol and vibrating platform of the present study [23]. However, no signs of discomfort during a single session of WBV $(15$ and $21 \mathrm{~Hz})$ during 10-min were observed in healthy adult horses [5], probably because the hooves decrease the pressure on the vibratory platform.

The SPV of the common carotid artery is defined as the blood flow reaching the brain, and the RI is the physical resistance of the vascular wall, being directly proportional to the PI and inversely proportional to the blood flow $[1,25]$. No significant variation of SPV, RI, and PI values were identified in both arteries of G1 and G2. However, a SPV increase, and PI and RI decrease were observed in both groups. The authors believed that WBV can be beneficial for the brain due to a possible increase in blood flow.

Studies regarding the effects of WBV in the dopplerfluxometry parameters were made in healthy dogs. The RI of the renal artery did not show significant variations after a single WBV session [10]. On the other hand, a significant increase of RI of the femoral artery was observed after single daily use of WBV for five consecutive days in dogs [23].

The limitations of this study were: a low number of subjects due to the rigorous inclusion criteria, a fact that could influence the absence of significant variation in the evaluated time-points, and the absence of a control group which composed by dogs that did not receive vibratory therapy. Dogs of both groups attended as their control by using the parameters before the WBV stimulus as a physiologic value. Further studies need to be done with more dogs and group which will not receive any mechanical vibration and evaluated the chronic effects of the WBV.

\section{CONCLUSION}

A single session of WBV (30 and $50 \mathrm{~Hz}$ did, for $15 \mathrm{~min}$ ) do not produce undesirable effects on SPV, $\mathrm{RI}$, and PI of both common carotid arteries in adult and elderly non-athletic healthy dogs.

\author{
MANUFACTURERS \\ ${ }^{1}$ Amira Balanças Ltd. São Paulo, SP, Brazil. \\ ${ }^{2}$ Maschinen GmbH. Heusenstamm, Germany. \\ ${ }^{3}$ TheraPlate. Weatherford, TX, USA. \\ ${ }^{4}$ Esaote Healthcare do Brasil. São Paulo, SP, Brazil. \\ ${ }^{5}$ GraphPad Instat. DataSet1.ISD. New York, NY, USA.
}

Acknowledgements. The authors thank the Brasilian National Council for Scientific and Technological (CNPq) (Grant $n^{\circ}$ 46721).

Ethical approval. This experiment was approved and performed under the guidelines of the Ethics Committee in Animal Experimentation of the Faculty of Veterinary Medicine and Animal Science (FMVZ) of UNESP - São Paulo State University, Botucatu, São Paulo, under protocol no 0133/2017.

Declaration of interest. The authors report no conflicts of interest. The authors alone are responsible for the content and writing of the paper.

\section{REFERENCES}

1 Barreto J.L.W., Silva B.M., Da Silva L.E., Nóbrega A.C.L. \& Thomaz T.G. 2010. Fluxos nas carótidas e artérias vertebrais extracranianas em pacientes com morte cerebral. Revista Brasileira de Cardiologia. 23(1): 57-67.

2 Bezuidenhout A. 2013. The Heart and Arteries. In: Evans H.E. \& Lahunta A. (Eds). Miller's Anatomy of the dog. 4th edn. St. Louis: Elsevier Saunders Co., pp.444-466.

3 Cardinale M. \& Bosco C. 2003. The use of vibration as an exercise intervention. Exercise and Sport Sciences Reviews. 31(1): 3-7. DOI: 10.1097/00003677-200301000-00002.

4 Cardinale M. \& Wakeling J. 2005. Whole body vibration exercise: are vibrations good for you? British Journal of Sports Medicine. 39(9): 585-589. DOI: 10.1136/bjsm.2005.016857.

5 Carstanjen B., Balali M., Gajewski Z., Furmanczyk K., Bondzio A., Remy B. \& Hartmann H. 2013. Short-term Whole-body vibration exercise in adult healthy horses. Polish Journal of Veterinary Sciences. 16(2): 403-405. DOI: 10.2478/pjvs-2013-0057. 
6 Cochrane D.J. 2011. Vibration exercise: the potential benefits. International Journal of Sports Medicine. 32(2): 75-99. DOI: $10.1055 / \mathrm{s}-0030-1268010$.

7 Delecluse C., Roelants M. \& Verschueren S. 2003. Strength increase after Whole-body vibration compared with resistance training. Medicine and Science in Sports and Exercise. 35(6): 1033-1041. DOI: 10.1249/01.MSS.0000069752.96438.B0.

8 Dolny D. G. \& Reyes G.F.C. 2008. Whole body vibration exercise: training and benefits. Current Sports Medicine Reports. 7(3): 152-157. DOI: 10.1097/01.CSMR.0000319708.18052.a1.

9 Franchignoni F., Vercelli S. \& Ozçakar L. 2013. Hematuria in a runner after treatment with Whole-body vibration: a case report. Scandinavian Journal of Medicine \& Science in Sports. 23(3): 383-385. DOI: 10.1111/j.16000838.2012.01478.x

10 Freire L., Rahal S.C., Dos Santos I.F.C., Teixeira C.R., Inamassu L.R. \& Mamprim M.J. 2015. Renal resistive index of adult healthy dogs submitted to short-term Whole-body vibration exercise. Asian Journal of Animal and Veterinary Advances. 10(11): 797-802. DOI: 10.3923/ajava.2015.797.802.

11 Haas C.T., Turbanski S., Kessler K. \& Schmidtbleicher D. 2006. The effects of random Whole-body-vibration on motor symptoms in Parkinson's disease. NeuroRehabilitation. 21(1): 29-36. PMID: 16720935.

12 Ishitake, T., Miyazaki Y., Ando H. \& Matoba T. 1999. Suppressive mechanism of gastric motility by Whole-body vibration. International Archives of Occupational and Environmental Health. 72(7): 469-474. DOI: 10.1007/s004200050400.

13 Jordan M.J., Norris S.R., Smith D.J. \& Herzog W. 2005. Vibration training: an overview of the area, training consequences, and future considerations. Journal of Strength and Conditioning Research. 19(2): 459-466. DOI: 10.1519/13293.1.

14 Laflamme D. 1997. Development and validation of a body condition score system for dogs. Canine Practice. 22(4): $10-15$.

15 Lau R.W.K., Liao L.-r., Yu F., Teo T., Chung R.C.K. \& Pang M.Y.C. 2011. The effects of Whole-body vibration therapy on bone mineral density and leg muscle strength in older adults: a systematic review and meta-analysis. Clinical Rehabilitation. 25(11): 975-988. DOI: 10.1177/0269215511405078.

16 Miyazaki Y. 2000. Adverse effects of Whole-body vibration on gastric motility. The Kurume Medical Journal. 47(1): 79-86. DOI: 10.2739/kurumemedj.47.79.

17 Monteleone G., De Lorenzo A., Sgroi M., De Angelis S. \& Di Renzo L. 2007. Contraindications for Whole-body vibration training: a case of nephrolithiasis. The Journal of Sports Medicine and Physical Fitness. 47(4): 443-445. PMID: 18091685.

18 Park S.-Y., Son W.-M. \& Kwon O.-S. 2015. Effects of Whole-body vibration training on body composition, skeletal muscle strength, and cardiovascular health. Journal of Exercise Rehabilitation. 11(6): 289-295. DOI: 10.12965/ jer. 150254.

19 Prisby R.D., Lafage-Proust M.H., Malaval L., Belli A. \& Vico L. 2008. Effects of Whole-body vibration on the skeleton and other organ systems in man and animal models: what we know and what we need to know. Ageing Research Reviews. 7(4): 319-329. DOI: 10.1016/j.arr.2008.07.004.

20 Rauch F., Sievanen H., Boonen S., Cardinale M., Degens H., Felsenberg D., Roth J., Schoenau E., Verschueren S. \& Rittweger J. 2010. Reporting Whole-body vibration intervention studies: recommendations of the international society of musculoskeletal and neuronal interactions. Journal of Musculoskeletal \& Neuronal Interactions. 10(3): 193198. PMID: 20811143.

21 Rittweger J. 2010. Vibration as an exercise modality: how it may work, and what its potential might be. European Journal of Applied Physiology. 108(5): 877-904. DOI: 10.1007/s00421-009-1303-3.

22 Santos I.F.C., Rahal S.C., Freire L., Teixeira C.R., Inamassu L.R., Mamprim M.J., Gomes M.V.F. \& Tannus F.C.I. 2017. Acute effect of Whole-body vibration in a female dog with metritis. Acta Scientiae Veterinariae. 45(Suppl 1): 185. DOI: 456/1679-9216.85344.

23 Santos I.F.C., Rahal S.C., Santos A., Inamassu L., Rodrigues M., Tsunemi M., Mamprim M., Rodrigues C., Teixeira C. \& Sato T. 2019. Whole-body vibration on leg muscles thermography and femoral resistive index of in adult healthy dogs. Research in Veterinary Science. 122: 118-123. DOI: 10.1016/j.rvsc.2018.11.003.

24 Santos I.F.C., Rahal S.C., Shimono J., Tsunemi M., Takahira R. \& Teixeira C.R. 2017. Whole-body vibration exercise on hematology and serum biochemistry in healthy dogs. Topics in Companion Animal Medicine. 32(2): 86-90. DOI: 10.1053/j.tcam.2017.07.006. 
25 Shimizu Y., Itoh T., Hougaku H., Nagai Y., Hashimoto H., Sakaguchi M., Handa N., Kitagawa K., Matsumoto M. \& Hori M. 2001. Clinical usefulness of duplex ultrasonography for the assessment of renal arteriosclerosis in essential hypertensive patients. Hypertension Research: Official Journal of the Japanese Society of Hypertension. 24(1): 13-17. DOI: 10.1291/hypres.24.13.

26 Turbanski S., Haas C.T., Schmidtbleicher D., Friedrich A. \& Duisberg P. 2005. Effects of random Wholebody vibration on postural control in Parkinson's disease. Research in Sports Medicine. 13(3): 243-256. DOI: $10.1080 / 15438620500222588$.

27 Van Nes I.J.W., Geurts A.C.H., Hendricks H.T. \& Duysens J. 2004. Short-term effects of Whole-body vibration on postural control in unilateral chronic stroke patients: preliminary evidence. American Journal of Physical Medicine \& Rehabilitation. 83(11): 867-873. DOI: 10.1097/01.phm.0000140801.23135.09.

28 Vilar J.M., Rubio M., Carrillo J.M., Domínguez A.M., Mitat A. \& Batista M. 2016. Biomechanic characteristics of gait of four breeds of dogs with different conformations at walk on a treadmill. Journal of Applied Animal Research. 44(1): 252-257. DOI: 10.1080/09712119.2015.1031778.

29 Yamada E., Takashi K., Miyamoto K., Tanaka S., Morita S., Tanaka S., Tsuji S., Mori S., Norimatsu H. \& Itoh S. 2005. Vastus lateralis oxygenation and blood volume measured by near-infrared spectroscopy during Whole-body vibration. Clinical Physiology and Functional Imaging. 25(4): 203-208. DOI: 10.1111/j.1475-097X.2005.00614.x. 